\title{
Gender Differences in Disease Activity and Impact in Axial Spondyloarthritis
}

\author{
Ying Ying Leung ${ }^{1}$ (D)
}

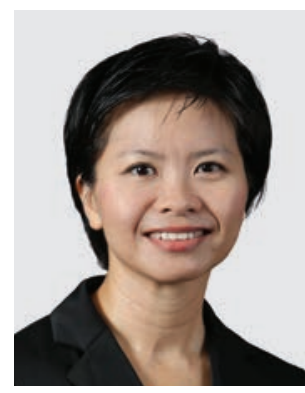

Ankylosing spondylitis (AS), characterized by inflammatory back pain and sacroiliitis on radiography, was traditionally considered a condition predominant in men. Since the introduction of the 2009 Assessment in Spondyloarthritis international Society classification criteria ${ }^{1}$ aiming to facilitate earlier classification of cases without radiographic sacroiliitis, more women have been classified as having axial spondyloarthritis (axSpA). The prevalence of men/women with AS, which was 3:1 in the $1990 \mathrm{~s},{ }^{2}$ is now equal in newer cohorts, particularly for nonradiographic (nr-) axSpA. ${ }^{3}$ Differences in the manifestations of axSpA between men and women have been increasingly recognized in the last decade. Studies have reported that women have more peripheral disease and higher pain, fatigue, and disease activity, whereas men have higher inflammatory markers and structural damage on radiography. ${ }^{4,5}$

In this issue of The Journal of Rheumatology, Mease, et al report the sex differences in axSpA using real-world data from the Corrona Registry. ${ }^{6}$ The Corrona Psoriatic Arthritis and Spondyloarthritis (PsA/SpA) Registry is a large prospective observational cohort started in 2013 from 58 practices across 30 states in the United States. In their study, 498 patients with rheumatologist-diagnosed axSpA (without concurrent diagnosis of PsA) with baseline data for sex were included. Of these, $191(38.4 \%)$ were women. There was a long delay in diagnosis of over 7 years in patients. In contrast to previous studies that cited a longer delay in diagnosis among women, ${ }^{7}$ there were no differences in diagnosis delay comparing men and women; this is possibly related to less emphasis on radiographic sacroi-

YYL is supported by the National Medical Research Council, Singapore (NMRC/CSA-Inv/0022/2017). The funding sources had no role in views expressed in this editorial.

${ }^{I}$ Y.Y. Leung, MBChB, MD, Department of Rheumatology and Immunology, Singapore General Hospital, and Duke-NUS Medical School, Singapore.

$Y Y L$ has received speaker fees from AbbVie, DKSH, Janssen, Novartis, and Pfizer.

Address correspondence to Dr. Y.Y. Leung, Department of Rheumatology and Immunology, Singapore General Hospital, The Academia, level 4, 20 College Road, Singapore 169856.Email:katyccc@hotmail.com. liitis in diagnosis in the last decade. Women had more peripheral musculoskeletal manifestations, including higher tender/ swollen joint counts and enthesitis count, and were more likely to have used prior conventional synthetic disease-modifying antirheumatic drugs and prednisolone. In addition, women had higher disease activity, poorer physical function, and worse physician global assessment compared to men. These differences between men and women were more prominent among patients with nr-axSpA than AS. Women had greater work productivity and activity impairment and were less likely to work full time than men. However, the higher rate of work productivity loss in women could be due to factors other than mere sex differences, including physical demand and the nature of the job. In addition, there may be social and cultural implications for men vs women in continuing or not continuing with full-time employment when facing a change in health condition.

There may be sex- or gender-related factors that could explain the variation in the manifestations between men and women with axSpA (Figure 1). Sex refers to a set of biological attributes related to chromosomes, genes and expression, hormonal function, and physique, whereas gender refers to the self-identity of men and women as shaped by psychological, social, and cultural influences.

Biological plausibility that may explain the sex-related differences in axSpA includes genetic, hormonal, and immunological aspects. The prevalence of HLA-B27, the most important genetic predisposition for axSpA, is higher among men with axSpA,${ }^{8,9}$ and partially explains the higher radiographic damage and the spine and sacroiliac joint inflammation on magnetic resonance imaging among men than women..$^{10}$ Further, other genes and single-nucleotide polymorphisms related to osteogenesis and ankylosis were found to be more prevalent in men than women. ${ }^{11,12}$ Hormonal differences may also contribute to the disparity in immune responses in men and women. Estrogen increases production of interleukin (IL)-1, IL-6, and tumor necrosis factor (TNF)- $\alpha,{ }^{13}$ whereas testosterone decreases TNF- $\alpha$ production but increases the production of antiinflammatory IL-10. ${ }^{14}$ Yet, conflicting results in studies in the role of

See Sex differences in axSpA, page 1528 


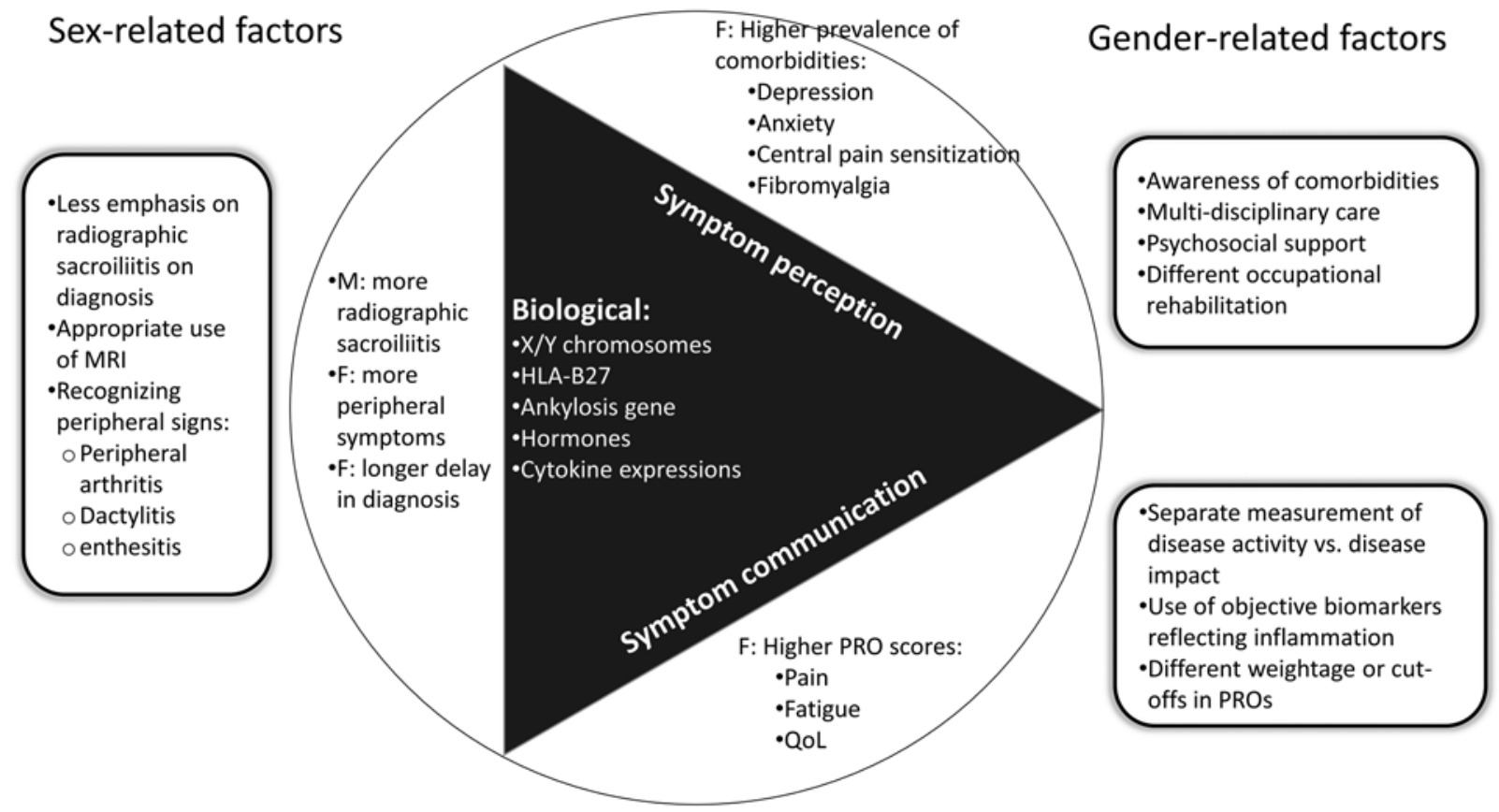

Figure 1. Sex-related and gender-related factors explaining differences in manifestations between men and women with axial spondyloarthritis. F: female; M: male; PRO: patient-reported outcome; MRI: magnetic resonance imaging; QoL: quality of life.

sex hormones in the development or progression of SpA warrant further investigation. From an immunological perspective, the frequency of peripheral blood IL-17A and Th17 cells, which are key players in the IL-23/IL-17 axis, was elevated in men but not in women with AS, compared to healthy controls..$^{15}$ Again, this highlights the differences of activation status of the immune system that may underlie the sex-related differences in manifestations in axSpA.

A large part of the observed differences between men and women with axSpA may be related to gender. Consistent with previous studies, Mease, et al observed higher disease activity among women compared to men, most prominently in patient-reported outcomes (PROs) such as the Bath AS Disease Activity Index. ${ }^{6}$ There was no statistically significant difference seen with the AS Disease Activity Score, whereas C-reactive protein (CRP) was comparable between sexes in the study. Other PROs, including pain, fatigue, and Health Assessment Questionnaire-Disability Index, were scored higher among women compared to men. Interestingly, when patients were asked to rate their global assessment of disease activity, there was no statistically significant difference in scores between men and women. The exact reasons why men and women may experience life impact of axSpA differently are not entirely understood. Intuitively, these contrasts could be related to both the experience of symptoms and the reporting of these symptoms as they conform to gender roles. Social responsibilities vary between genders and so do expectations; therefore, there are also distinct ways that men and women may communicate and report those symptoms according to social norms. For example, women generally report higher pain intensity compared to men across both musculoskeletal and nonmusculoskeletal conditions. ${ }^{16}$ However, a major contributing factor in axSpA could be central pain sensitization, which refers to the amplification of neural signaling within the central nervous system that leads to pain hypersensitivity and the spread of pain to a broader area than the primary inflammatory sites. A similar concept is found in fibromyalgia (FM), which encompasses widespread pain, fatigue, sleep disturbance, and cognitive symptoms, and is a well-known contextual factor associated with overestimating measurements of disease activity. ${ }^{17}$ Using a body chart for painful locations in the body, it has been shown previously that women with axSpA have a more widespread pattern of painful locations, both along the axial and peripheral articular areas, than men. ${ }^{18}$ In the Corrona Registry, Mease, et al collected data for FM according to physicians' judgment. ${ }^{6}$ The overall prevalence of FM (4.8\%) was at the low end among those reported for $\mathrm{SpA},{ }^{19}$ yet there was a remarkable disparity in the prevalence of FM between sexes in the study (1\% in men vs $10.5 \%$ in women). A higher prevalence of physician-reported depression among women compared to men was also observed ( $12.1 \%$ in men vs $25.7 \%$ in women), highlighting the psychological comorbidities that may influence PROs.

This study highlighted several important points. First, women with axSpA have more peripheral disease burden, the awareness of which should assist in earlier diagnosis. Second, disease activity measurements relying heavily on PROs are more vulnerable to contextual factors, such as comorbid FM and depression. Incorporating objective measurement of the pathophysiological inflammatory process is desirable. CRP could be an option, and the development of more objective biomarkers 
is an important unmet need. Third, the life impact of SpA may be experienced differently for men and women. Awareness of the sex differences in disease impact is relevant to facilitate early diagnosis of axSpA, assessment of disease activity in axSpA, and personalization of treatment strategies for patients with distinct healthcare needs.

\section{REFERENCES}

1. Rudwaleit M, van der Heijde D, Landewe R, Listing J, Akkoc N, Brandt J, et al. The development of Assessment of SpondyloArthritis international Society classification criteria for axial spondyloarthritis (part II): validation and final selection. Ann Rheum Dis 2009;68:777-83.

2. Kennedy LG, Will R, Calin A. Sex ratio in the spondyloarthropathies and its relationship to phenotypic expression, mode of inheritance and age at onset. J Rheumatol 1993;20:1900-4.

3. Sieper J, van der Heijde D. Review: nonradiographic axial spondyloarthritis: new definition of an old disease? Arthritis Rheum 2013;65:543-51.

4. Wright GC, Kaine J, Deodhar A. Understanding differences between men and women with axial spondyloarthritis. Semin Arthritis Rheum 2020;50:687-94.

5. Rusman T, van Bentum RE, van der Horst-Bruinsma IE. Sex and gender differences in axial spondyloarthritis: myths and truths. Rheumatology 2020;59 Suppl 4:iv38-46.

6. Mease PJ, McLean RR, Dube B, Liu M, Rebello S, Glynn M, et al. Comparison of men and women with axial spondyloarthritis in the US-based Corrona Psoriatic Arthritis/Spondyloarthritis registry. J Rheumatol 2021;48:1528-36.

7. Jovani V, Blasco-Blasco M, Ruiz-Cantero MT, Pascual E. Understanding how the diagnostic delay of spondyloarthritis differs between women and men: a systematic review and metaanalysis. J Rheumatol 2017;44:174-83.

8. Tournadre A, Pereira B, Lhoste A, Dubost JJ, Ristori JM, Claudepierre P, et al. Differences between women and men with recent-onset axial spondyloarthritis: results from a prospective multicenter French cohort. Arthritis Care Res 2013;65:1482-9.
9. Ortolan A, van Lunteren M, Ramiro S, Ramonda R, Landewe RBM, Dagfinrud H, et al. Are gender-specific approaches needed in diagnosing early axial spondyloarthritis? Data from the SPondyloArthritis Caught Early cohort. Arthritis Res Ther 2018;20:218.

10. Chung HY, Machado P, van der Heijde D, D’Agostino MA, Dougados M. HLA-B27 positive patients differ from HLA-B27 negative patients in clinical presentation and imaging: results from the DESIR cohort of patients with recent onset axial spondyloarthritis. Ann Rheum Dis 2011;70:1930-6.

11. Tsui HW, Inman RD, Paterson AD, Reveille JD, Tsui FW. ANKH variants associated with ankylosing spondylitis: gender differences. Arthritis Res Ther 2005;7:R513-25.

12. Tsui HW, Inman RD, Reveille JD, Tsui FW. Association of a TNAP haplotype with ankylosing spondylitis. Arthritis Rheum. 2007;56:234-43.

13. Fish EN. The X-files in immunity: sex-based differences predispose immune responses. Nat Rev Immunol. 2008;8:737-44.

14. Mohamad NV, Wong SK, Wan Hasan WN, Jolly JJ, Nur-Farhana MF, Ima-Nirwana $S$, et al. The relationship between circulating testosterone and inflammatory cytokines in men. Aging Male 2019;22:129-40.

15. Gracey E, Yao Y, Green B, Qaiyum Z, Baglaenko Y, Lin A, et al. Sexual dimorphism in the Th17 signature of ankylosing spondylitis. Arthritis Rheumatol 2016;68:679-89.

16. Ruau D, Liu LY, Clark JD, Angst MS, Butte AJ. Sex differences in reported pain across 11,000 patients captured in electronic medical records. J Pain 2012;13:228-34.

17. Leung YY, Thumboo J. Fibromyalgia as a contextual factor influencing disease activity measurements in spondyloarthritis and psoriatic arthritis. J Rheumatol 2016;43:1953-5.

18. Swinnen TW, Westhovens R, Dankaerts W, de Vlam K. Widespread pain in axial spondyloarthritis: clinical importance and gender differences. Arthritis Res Ther 2018;20:156.

19. Mease PJ. Fibromyalgia, a missed comorbidity in spondyloarthritis: prevalence and impact on assessment and treatment. Curr Opin Rheumatol 2017;29:304-10. 